\title{
Transmission Line Traveling Wave Fault Location Based on Empirical Mode Decomposition De-noising
}

\author{
Shan Hong ${ }^{1}$, Baohua Wang ${ }^{1, *}$, Xiaodong Liu ${ }^{2}$ \\ ${ }^{1}$ Nanjing University of Science and Technology, Nanjing, China \\ ${ }^{2}$ Nanjing Power Supply Company, Nanjing, China \\ ${ }^{*}$ Corresponding author
}

\begin{abstract}
Wavelet analysis and detection of modulus maximum are usually used to extract fault wave head. But for the actual transmission line, the existence of noise will affect the accurate extraction of wave head, leading to failure of location. To avoid the serious distortion caused by directly removing high frequency components of signal, a new de-noising method based on empirical mode decomposition is proposed. The actual recorded data of a transformer substation were analyzed with matlab. The results of wavelet analysis to noise signal and de-noised signal are compared to verify the feasibility and practicability of this method.
\end{abstract}

Keywords-empirical mode decomposition; threshold de-noising; traveling wave fault location; wavelet analysis

\section{INTRODUCTION}

With the development of electric power system, voltage level and transmission capacity of transmission line are gradually increasing, and the impact of transmission line fault on social and economic life has become more and more. When transmission line fault occurred, it is necessary to determine the position of fault and restore the power supply quickly and accurately, which is the key link to ensure the reliability of power system.

Traveling wave fault location is based on the consideration of transmission line distributed parameter, directly use transient traveling wave signal generated by fault point to calculate and analyze. It has the advantage of not being affected by factors like operation mode, fault type and transition resistance [1]. It includes single and double terminal fault location method [2].

Single terminal traveling wave fault location method will not be affected by length of line and time error of timing system; can provide more accurate measurement results and stronger real-time characteristics than the double terminal method.

Traditional traveling wave fault location in time domain often does not consider the defects that the existence of noise in actual signal will cause interference and the uncertainty of wave velocity and line sag will affect the accuracy of location. The traditional de-noising method based on wavelet decomposition directly filters out the high-frequency components, which leads to serious distortion. Moreover, this method strongly dependents on wavelet base selection and has poor adaptability.
A single terminal location method to avoid the influence of wave speed is proposed in [3], which effectively solves the problem of location accuracy caused by the uncertainty of wave velocity at different voltage levels and transmission line sag.

According to the analysis above, a transmission line traveling wave fault location method based on empirical mode decomposition (EMD) de-noising is proposed. Firstly, the initial signal is decomposed by EMD. Then, the first few highfrequency IMF components respectively are threshold filtered. The desired signal is reconstructed by adding the filtered IMF and unfiltered IMF. Finally, using the actual recorded data of a transformer substation, the results of wavelet analysis to noise signal and de-noised signal are compared to verify the feasibility and practicability of this method.

\section{PRICIPLE OF EMD DENOISING}

\section{A. The Basic Principle of EMD}

The essence of EMD is processing a complex signal smoothly. Signal's fluctuations or trends in different scales gradually are decomposed into a plurality of stationary components and a residual component [4-6]. Stationary components called intrinsic mode function (IMF) represent the different scale characteristics of signal. Residual component represents the trend of the signal.

Not all decomposed components are IMF components, only if the components comply with the following two conditions [7]:

1) In entire data sequence, the number of local extreme points and zero-crossings of function must be equal, or at most differ by only one;

2) At any schedule point, the average value of local maximum envelope (upper envelope) and local minimum envelope (lower envelope) must be zero.

The first condition is obvious, which is similar to Gaussian stationary process narrowband requirement. The second condition is its innovation, and its essence is "local means of data is zero", which change the traditional global limited to locality limited. For non-stationary signal, it is difficult to define and calculate the local mean, so local mean is substituted for the average of local maximum envelope and local minimum envelope to make the signal waveform partial symmetrical. 
Process of empirical mode decomposition is shown in Figure 1.

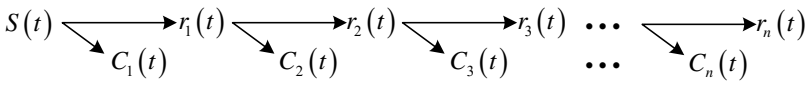

$$
\begin{aligned}
& \text { FIGURE I. PROCESS OF EMD }
\end{aligned}
$$

Initial signal $S(t)$ can be expressed as:

$$
S(t)=\sum_{i=1}^{n} C_{i}(t)+r_{n}(t)
$$

Where: $C_{i}(t)$ represents the $i$-th IMF; $r_{n}(t)$ represents residual component, usually indicates the DC component.

EMD algorithm flow diagram is shown in Figure 2.

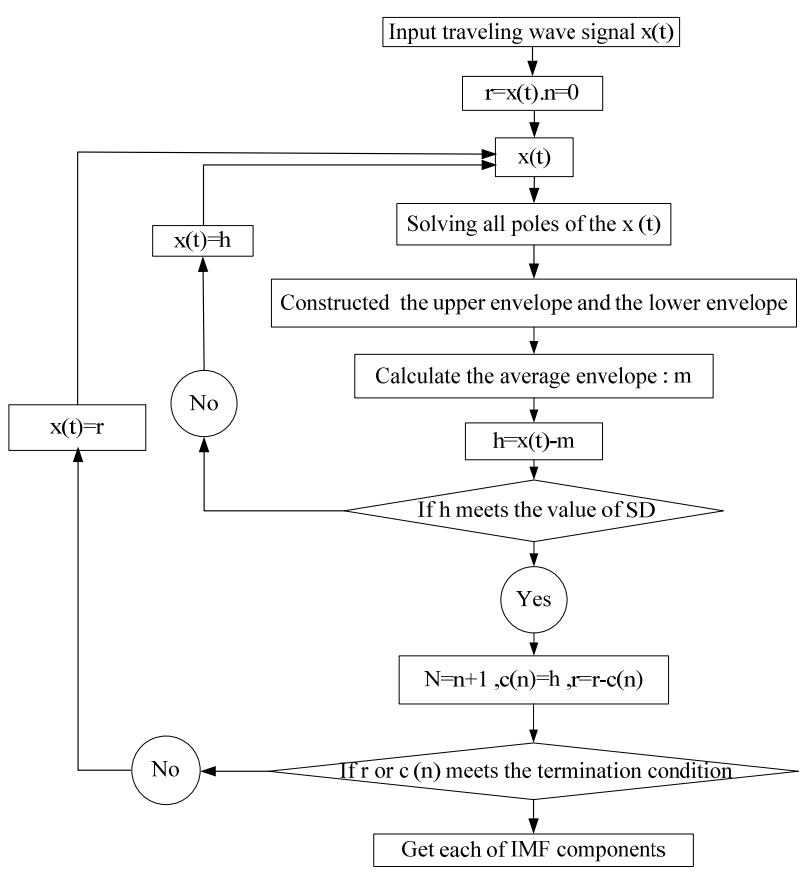

FIGURE II. FLOW DIAGRAM OF EMD ALGORITHM

\section{B. Thershold De-noising Based on EMD}

EMD method has good characteristics, which draws on the advantages of wavelet transform: multi-resolution, and also overcomes the difficulty of choosing the wavelet base [8]. EMD is a process filtering continuously from low frequency to high frequency. Each IMF component contains a range of characteristic scale This feature can be used to construct a new filter [9-10].Different with the traditional filter, it is not based on the frequency domain, but based on local characteristic time scale parameter, which does not require to specify center frequency, bandwidth and other parameters humanly, also does not need to specify wavelet type and decomposition layer.

Since the frequency of each IMF component almost decrements by 2 negative power and the frequency of noise is high, de-noising can be achieved simply by subtracting the high frequency components from the original noisy signal [11]. But for the circumstance that noise and useful signal frequency aliasing in IMF components, using such method directly will lead to useful signal in IMF components is filtered with noise together, causing serious distortion of the signal [12].Therefore, a threshold de-noising method based on EMD is proposed; Figure 3 is the block diagram of EMD threshold de-noising.

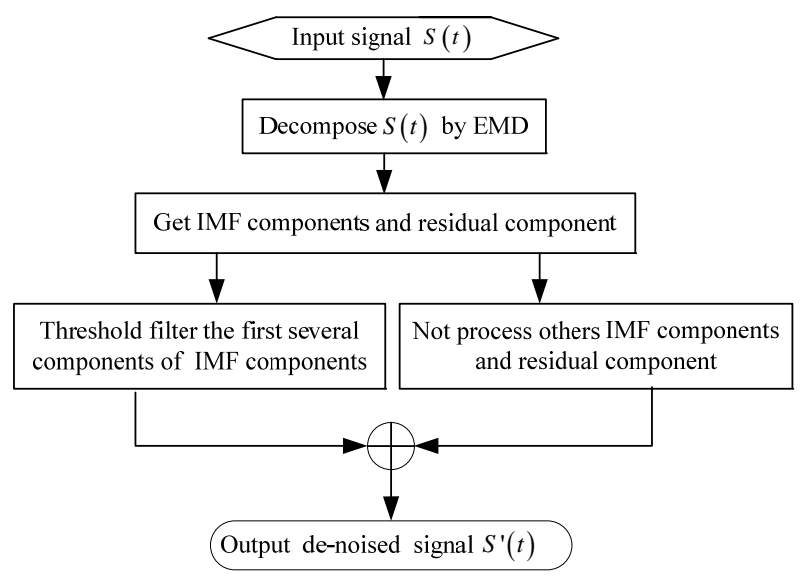

FIGURE III. BLOCK DIAGRAM OF EMD THRESHOLD DE-NOISING

\section{APPLICATION OF EMD IN TRAVELING WAVE FAUlT LOCATION}

\section{A. Single Terminal Location Principle}

Traditional traveling wave fault location methods must combine initial fault traveling wave with velocity of wave to measure fault distance. However, the wave velocity is essentially an uncertain quantity in the propagation process, which will cause a great impact on the accuracy of traveling wave fault location.

Traveling wave propagation process is shown in Figure 4.In the figure, $\mathrm{M}, \mathrm{N}$ respectively represent two ends of the bus, $\mathrm{F}$ is the fault point, $t_{1}$ is the absolute time that fault initial traveling wave reached to the $\mathrm{M}$ end bus, $t_{2}$ is the absolute time that the reflected wave of the fault point reached to the $\mathrm{M}$ end bus, $t_{3}$ is the absolute time that the reflected wave of pair end bus reached to the $\mathrm{M}$ end bus.

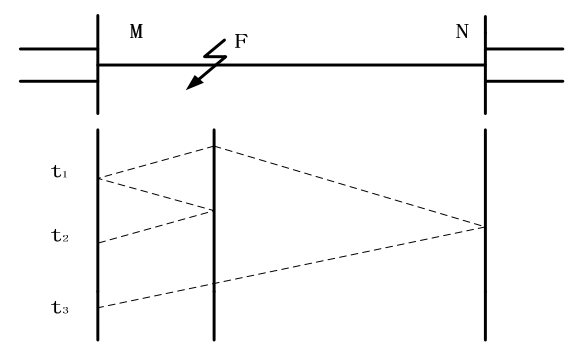

FIGURE IV. GRID CHART OF LOCATION TRAVELING WAVE

According to figure 4 yields the following equation: 


$$
\left\{\begin{array}{l}
v\left(t_{1}-t_{0}\right)=x \\
v\left(t_{2}-t_{0}\right)=3 x \\
v\left(t_{3}-t_{0}\right)=2 l-x
\end{array}\right.
$$

Eliminating the velocity $v$ in (2), equation of location that avoiding the impact of wave velocity is shown in (3).

$$
\left\{\begin{array}{l}
t_{0}=\left(3 t_{1}-t_{2}\right) / 2 \\
x=\left(t_{2}-t_{1}\right) l /\left(t_{3}-2 t_{1}+t_{2}\right)
\end{array}\right.
$$

Where $l$ is the line length, $x$ is the actual distance from $\mathrm{F}$ to the $\mathrm{M}$ end bus; $t_{0}$ is the absolute time when fault occurred.

\section{B. Process of Traveling Wave Fault Location}

Above discussion is carried out under ideal conditions, namely without considering the actual lines are always more or less affected by the white noise. The existence of noise will impact the accurate extraction of fault traveling wave head, coupled with the attenuation of signal by external branch, reflected waves of fault point may be completely submerged in the noise signal .Not being able to separated effectively the reflected wave of fault point from the noise will directly lead to the failure of location. Therefore, threshold de-noising based on EMD is used to modulus components.

Process of traveling wave fault location theory is shown in Figure 5.

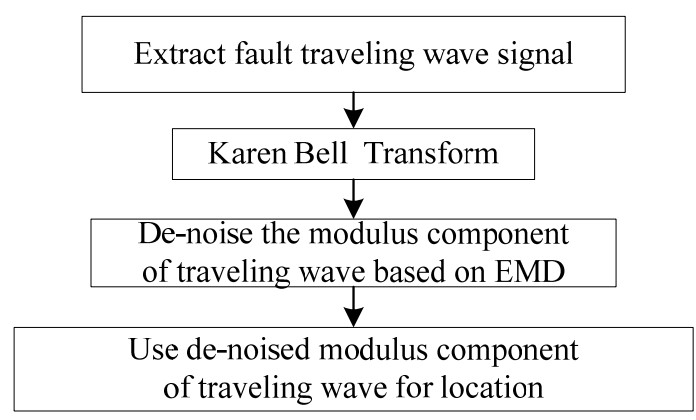

FIGURE V. PROCESS OF TRAVELING WAVE FAULT LOCATION THEORY

\section{APPLICATION EXAMPLE}

\section{A. Transmission Line Fault Reporting Description}

$\mathrm{AB}$ phase short circuit fault occurs to one transmission line of $500 \mathrm{kV}$ substation. Protection device operated correctly. The line length is $39.2 \mathrm{~km}$. The fault location result given by the protection device is $23.1 \mathrm{~km}$.

\section{B. Analysis of Fault Location Simulation}

CAAP2008X is cross-platform power fault recording analysis software, complete three-phase voltage and current waveforms recorded by CAAP2008X are shown in Figure 6.

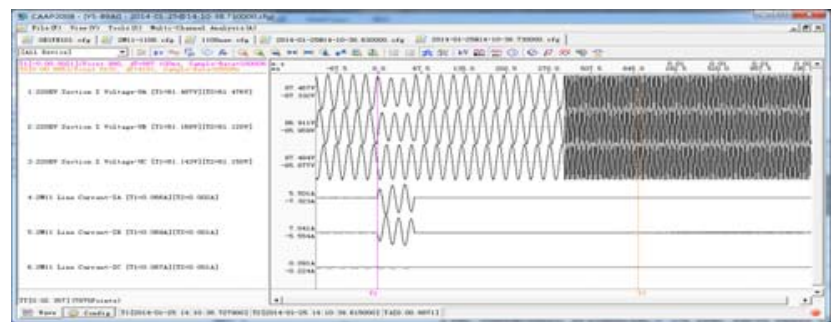

FIGURE VI. WAVEFORM RECORDED BY CAAP2008X

The sampling frequency of the first half of the waveform is $10000 \mathrm{~Hz}$, the latter part is $2000 \mathrm{~Hz}$. The oscillography in Figure 6 is cut out to obtain the current waveform of $0.09 \mathrm{~s} \sim 0.17 \mathrm{~s}$, as is shown in Figure 7.

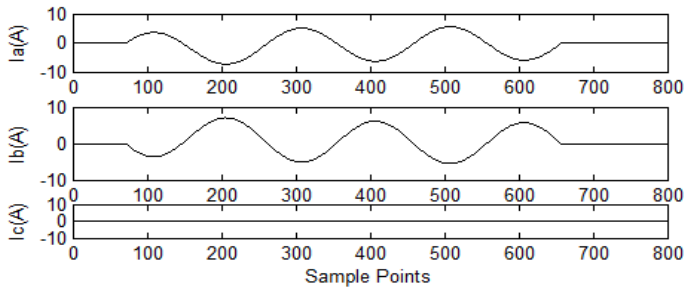

FIGURE VII. THREE-PHASE CURRENT WAVEFORM

For the 800 sampling points, the phase mode transformation is performed according to the Karen Bell transform:

$$
I_{\alpha}(n)=\frac{1}{3}\left[I_{a}(n)-I_{b}(n)\right]
$$

The modulus current $I_{\alpha}$, the current waveform is shown in Figure 8.

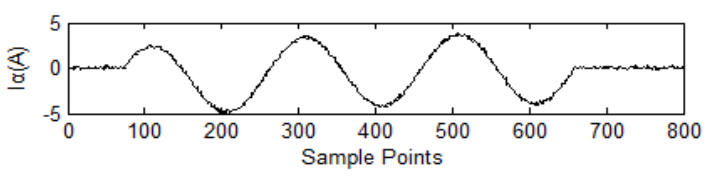

FIGURE VIII. INITIAL MODULUS CURRENT WAVEFORM

We can see that the modulus current contains noise, so decompose the signal using EMD. The result of EMD is shown in Figure 9.

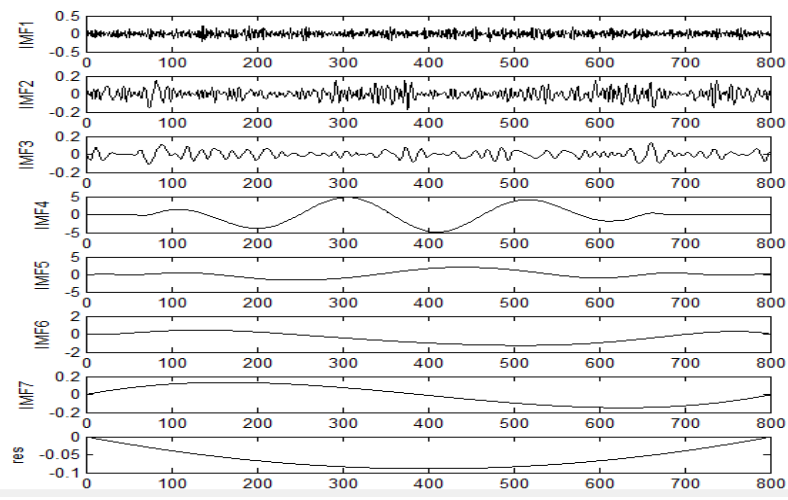

FIGURE IX. RESULT OF EMD 
Threshold filter IMF1 IMF4 component respectively and reconstruct the signal. Modulus current waveform denoising by EMD is shown in Figure 10.

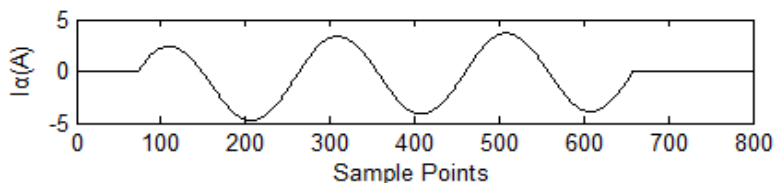

FIGURE X. MODULUS CURRENT WAVEFORM DE-NOISED BY EMD

Decompose initial modulus current and de-noised modulus current for five layers using $\mathrm{db} 4$ wavelet, the results are shown in Figure 11, Figure 12.

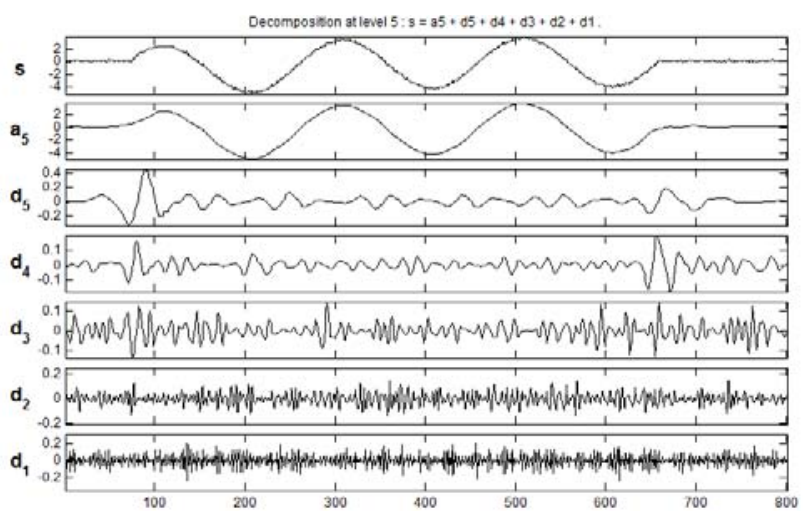

FIGURE XI. WAVELET DECOMPOSITION RESULT OF INITIAL MODULUS CURRENT WAVEFORM

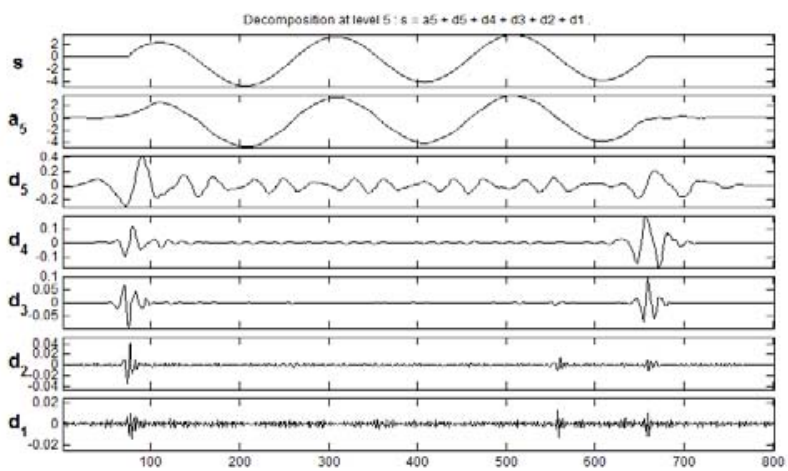

FIGURE XII. WAVELET DECOMPOSITION RESULT OF DE-NOISED MODULUS CURRENT WAVEFORM

According to Figure 11, the existence of noise has a great impact on the accurate extraction of fault traveling wave head. The result of fault location is $12.65 \mathrm{~km}$, which is seriously inconsistent with result of fault report, directly leading to the failure of fault location.

Observing the modulus maximum points of each scale in Figure 12, apparently time of the first wave head is the absolute time of fault initial traveling wave arrived to the $\mathrm{M}$ end bus.

The fault initial traveling wave has same polarity with the reflected wave of the fault point, and has opposite polarity with the reflected wave of pair end bus, so the third modulus maximum point represents the time that reflected wave of pair end bus arrive at $\mathrm{M}$ end bus, the second modulus maximum point represents the time that reflected wave of pair end bus reaches to $\mathrm{M}$ end bus. Namely, $t_{1}=78 \mu \mathrm{s}, t_{2}=241 \mu \mathrm{s}, t_{3}=191 \mu \mathrm{s}$. According to (3), $x=23.151 \mathrm{~km}$. It is quite close to the result of fault report: $23.1 \mathrm{~km}$.

The comparison of two results above shows that the EMD threshold de-noising can effectively remove the noise and improve the location accuracy.

\section{CONCLUSIONS}

Aimed at the existence of noise in the actual transmission line, this paper proposes a transmission line location method based on EMD de-noising. Using actual recorded data of a transformer substation, the results of wavelet analysis to noise signal and de-noised signal are compared. The comparison shows that the threshold de-noising based on EMD can effectively remove the noise in the signal. It proves that this method can be used for fault location in practical engineering, can improve the accuracy and feasibility of fault location.

\section{REFERENCES}

[1] B. Y. Xu, J. Li, P. Chen, "Modern fault location technoques based on fault generated traveling wave and their applications," Automation of Electric Power Systems, vol. 25(23) , pp. 62-65, 2001.

[2] J. Qin, X. X. Chen, J. C. Zheng, "Study on dispersion of traveling wave in transmission line," Proceedings of the Chinese Society of Electrical Engineering, vol. 19(9), pp. 27-30, 1999.

[3] T. Jiang, Y. P. Lu, "Study on transmission line singal terminal traveling wave fault location avoiding wave speed influence," Electric Power Automation Equipment, vol. 24(12), pp. 29-32, 2004.

[4] Y. Gan, L. Sui, J Wu, "An EMD threshold de-noising method for inertial sensors," Measurement, vol. 49(1), pp. 34-41, 2014.

[5] L. Li , X. Chai , S. Zheng, "A de-noising method for track state detection signal based on the statistical characteristic of noise," Journal of Transportation Technologies, vol. 04, 2014.

[6] L. Y. Sun, X. X. Huang, W. Cai, “An EMD-based signal de-noising method for ultrasonic pressure measurement outside pipeline," Technical Acoustics, 2011.

[7] N. E. Huang, Z. Shen, S. R. Long, "The empirical mode decomposition and the Hilbert spectrum for nonlinear and non-stationary time series analysis," Proceedings of the Royal Society of London (Series A): Mathematical, Physical and Engineering Sciences, vol. 454(1971), pp. 903-995, 1998.

[8] B. B. Cui, X. Y. Chen, "Multipath mitigation of GPS signal based on improved EMD algorithm," Journal of Chinese Inertial Technology, vol. 22(3), pp. 346-351, 2014

[9] Ahn J H, "Fault detection of a roller-bearing system through the EMD of a wavelet de-noised signal," Sensors, vol. 14(8), pp. 15022-15038, 2014.

[10] Y. X. Liu, J. J. Liu, Y. Y. Cao, “An improved method for wavelet threshold de-noising," Journal of Hebei North University, 2012.

[11] L. Li, X. Chai, S. Zheng, "A de-noising method for track state detection signal based on EMD," Journal of Signal \& Information Processing, vol. 5(4), pp. 104-111, 2014.

[12] J. F. Zhang, W. K. Chu, Q. Yao, "The design based on mathematical morphology and wavelet theory of fault location system on power cables," 2011 International Conference on. IEEE, pp. 1521-1524, 2011. 